\title{
Theory of near-gap second harmonic generation in centrosymmetric magnetic semiconductors: Europium chalcogenides
}

\author{
A. B. Henriques \\ Instituto de Física, Universidade de São Paulo, Caixa Postal 66318, 05315-970 São Paulo, Brazil \\ E. Abramof and P. H. O. Rappl \\ LAS-INPE, Av. dos Astronautas, 1758-Jd. Granja, 12227-010 São José dos Campos, Brazil \\ (Received 9 June 2009; published 9 December 2009)
}

\begin{abstract}
Second harmonic generation is strictly forbidden in centrosymmetric materials, within the electric dipole approximation. Recently, it was found that the centrosymmetric magnetic semiconductors EuTe and EuSe can generate near-gap second harmonics, if the system is submitted to an external magnetic field. Here, a theoretical model is presented, which well describes the observed phenomena. The model shows that second harmonic generation becomes efficient when the magnetic dipole oscillations between the band-edge excited states of the system, induced by the excitation light, enter the in-phase regime, which can be achieved by applying a magnetic field to the material.
\end{abstract}

DOI: $10.1103 /$ PhysRevB.80.245206

PACS number(s): 75.50.Pp, 42.65.Ky, 78.20.Ls

\section{INTRODUCTION}

Europium chalcogenides ( $\mathrm{Eu} X$, where $X=\mathrm{O}, \mathrm{S}, \mathrm{Te}$, or Te) are intrinsic magnetic semiconductors that have unique magneto-optical properties, which have attracted continuous attention ever since such pure compounds became available in the middle of the 20th century. The very large $S=7 / 2$ spin stored at each crystal lattice site occupied by Eu, combined with a transparency window in the visible range of the electromagnetic spectrum, motivated investigators to employ $\mathrm{Eu} X$ in magneto-optical devices. ${ }^{1}$ In more recent years, with the advent of spintronics, highly efficient spin filters based on $\mathrm{Eu} X$ were developed. ${ }^{2}$ It has also been demonstrated that $\mathrm{EuX}$ can be integrated into silicon technology, ${ }^{3}$ which enhances still further the prospects of employing $\mathrm{Eu} X$ in future spintronic devices.

In order to employ $\mathrm{Eu} X$ in specific devices, it is beneficial to have an understanding of their electronic energy structure, and a good understanding of the optical band-edge states has been gained over the years. ${ }^{4-10}$ Nonlinear optics is an important tool to investigate the electronic structure, because such effects as resonant multiple-order harmonic generation result in sharp spectral features that furnishes detailed information on the energy states accessible to the system under investigation, ${ }^{11}$ as well as unique and fascinating imaging of magnetic domains in solid-state crystals. ${ }^{12}$ The nonlinear optical properties of $\mathrm{Eu} X$ have been studied recently, ${ }^{13}$ and second (and third) harmonic generations were observed when the sample was submitted to a high magnetic field. This, at a first glance, seemed surprising, because electric dipole second-order harmonic generation (SHG) is strictly forbidden in centrosymmetric materials such as $\mathrm{Eu} X$; moreover, for $m 3 m$ symmetry, which applies to $\mathrm{Eu} X$, magnetic dipole induced SHG vanishes. ${ }^{14}$ The purpose of this paper is to develop a SHG theoretical model in $\mathrm{Eu} X$ based on the wellestablished band-edge states of $\mathrm{Eu} X$.

\section{MODEL}

In order to describe possible SHG mechanisms in europium chalcogenides, we introduce very briefly SHG theory applied to the specific scenario peculiar to $\mathrm{EuX}$. Let us consider an ensemble of identical independent centrosymmetric electron systems of concentration $N$, each of which is described by a Hamiltonian $\mathcal{H}_{0}$, with an energy spectrum $E_{a}, E_{b}, \ldots$ and corresponding wave functions $|a\rangle,|b\rangle, \ldots$ The interaction between each electron system and a monochromatic electromagnetic plane wave traveling along $z$ with wave vector $k_{z}$, and polarization vector $\hat{x}$, can be described by the interaction Hamiltonian

$$
V^{x}=\frac{e}{m_{0}} \boldsymbol{A} \cdot \boldsymbol{p},
$$

where the vector potential is given by $\boldsymbol{A}=\hat{x} A_{1}(z) e^{-i \omega t}+$ c.c., $A_{1}(z)=\frac{E_{x}}{2 i \omega} e^{i k_{z} z}$, and $E_{x}$ is the amplitude of the electric field in the light wave. Using the expansion

$$
e^{i k_{z} z}=1+i k_{z} z+\cdots,
$$

and keeping only the first two terms, we obtain ${ }^{15}$ the matrix elements $V_{a b}^{x}$ that will be needed below for the induced oscillating polarization calculation

$$
\begin{aligned}
V_{a b}^{x}= & \frac{e}{m_{0}}\left\langle a\left|A_{1}(z) p_{x}\right| b\right\rangle=\frac{e E_{a b} E_{x}}{2 \hbar \omega}\langle a|x| b\rangle+i \frac{e E_{a b} k_{z} E_{x}}{4 \hbar \omega}\langle a|z x| b\rangle \\
& +\frac{e B_{y}}{4 m_{0}}\left\langle a\left|L_{y}\right| b\right\rangle,
\end{aligned}
$$

where $E_{a b}=E_{a}-E_{b}, B_{y}$ is the amplitude of the magnetic field wave in the incoming light, and $L_{y}$ is the $y$ component of the angular momentum operator. The first, second, and third terms are denominated electric dipole (ED), electric quadrupole (QD), and magnetic dipole (MD) matrix elements between states $|a\rangle$ and $|b\rangle$, respectively.

Using Eq. (1) as a perturbation of $\mathcal{H}_{0}$, we can apply standard density-matrix theory to calculate the time-dependent second-order dipole moment that light induces in the electronic system. ${ }^{16,17}$ Suppose that the system under consideration is characterized by an energy gap, $E_{G}$, between the ground state and the excited ones, which are closely spaced 
together, i.e., the energy of the excited states $|m\rangle$ is much larger than difference in energy between them. Then for photon energies of about $\hbar \omega \sim \frac{1}{2} E_{G}$, the main contribution to the second-order-induced polarization will come from terms in which the difference $E_{m g}-2 \hbar \omega$ figures in the denominator. Therefore, we may discard the small antiresonant contributions $^{16}$ to the induced second-order dipole moment. Additionally, we shall assume that in our centrosymmetric system all excited states involved in the SHG process have the same parity, and an opposite parity in respect to the ground state. Therefore the electric dipole matrix elements between excited states vanishes. We also assume $T=0 \mathrm{~K}$, meaning that every electronic system is in the ground state $|g\rangle$ of energy $E_{g}$. Under these assumptions, the component of the second-order polarization in the direction of the polarization vector of the exciting light is found to be

$$
P_{x}^{(2)}(2 \omega)=P_{x 0}^{(2)}(2 \omega) e^{-i 2 \omega t}+\text { c.c. },
$$

where the complex amplitude of polarization is given by

$$
P_{x 0}^{(2)}(2 \omega)=N \sum_{m n} \frac{\mu_{g n}^{x} V_{n m}^{x} V_{m g}^{x}}{\left(E_{m g}-\hbar \omega+i \Gamma_{n g}\right)\left(E_{n g}-2 \hbar \omega+i \Gamma_{m g}\right)},
$$

$\mu_{g n}^{x}=e\langle g|x| n\rangle, x$ is the $x$ coordinate of the electron, $V_{n m}^{x}$ is given by the MD and QD terms in Eq. (3), $V_{m g}^{x}$ is given by the ED term in Eq. (3), and $\left(T_{2}\right)_{a b}=\hbar / \Gamma_{a b}$ is a characteristic relaxation time between states $|a\rangle$ and $|b\rangle .{ }^{16,17}$

\section{ELECTRONIC STATES IN EUROPIUM CHALCOGENIDES}

In the ground state of $\mathrm{Eu} X$, there are seven electrons with parallel spins in the $4 f$ shell of the $\mathrm{Eu}^{2+}$ ions in the crystal, leading to a total spin $S=7 / 2$ and angular momentum $L=0$. Thus the ground state can be represented in standard spectroscopic notation by the term symbol ${ }^{8} S_{7 / 2}$. The seven $4 f$ electrons that compose the ${ }^{8} S_{7 / 2}$ state are strongly localized at the Eu lattice sites, due to the strong nuclear Coulomb attraction, and shielded from the environment by electrons occupying $5 s$ and $5 p$ orbitals, therefore they can be described by atomic orbitals that are unaffected by the rest of crystal. In the lowest-energy excited states, one electron from the $\mathrm{Eu}^{2+}$ is transferred to the lowest-energy conduction band, which is built from $5 d$ orbitals of the $\mathrm{Eu}$ atom. The crystal field splits the $5 d$ orbitals into a lower-energy threefolddegenerate $5 d\left(t_{2 g}\right)$ state and a twofold-degenerate $5 d\left(e_{g}\right)$ state of an energy that is more than $1 \mathrm{eV}$ greater. ${ }^{8}$ Due to the resonance between $5 d\left(t_{2 g}\right)$ orbitals at neighboring lattice sites, the $5 d\left(t_{2 g}\right)$ states broaden into the lowest-energy conduction band in $\mathrm{EuX}$. Optical absorption measurements have shown that this conduction band can be described in the framework of tight-binding theory, ${ }^{18}$ and in EuTe displays an energy width of about $100 \mathrm{meV}$ width, and whose basis states are built from the $5 d\left(t_{2 g}\right)$ orbitals $\left(d_{x y}, d_{y z}\right.$, or $\left.d_{z x}\right) .^{10}$ The electronic states belonging to the $5 d\left(t_{2 g}\right)$ conduction band are henceforward denoted by $X$. Once an electron is removed from the ground state, the orbital momentum quantum number of the $\mathrm{Eu}$ atom becomes $L=3$, and the spin quantum number becomes $S=3$. Therefore a strong spinorbit interaction, which was absent in the ${ }^{8} S_{7 / 2}$ ground state, is switched on for the six electrons remaining in the $4 f$ shell. The new quantum numbers that describe the stationary states of the $\mathrm{Eu}^{3+}$ remainder become the orbital momentum quantum number $L=3$, the spin quantum number $S=3$, and total momentum quantum number $J=0, \ldots, 6$, therefore the spectroscopic notation for the $\mathrm{Eu}^{3+}$ core is ${ }^{7} F_{J}$. Additionally the projection $M=-J, \ldots,+J$ of the total momentum of the $\mathrm{Eu}^{3+}$ spins on a quantization axis is defined, thus the complete specification of the lowest-energy excited states of $\mathrm{Eu} X$ can be represented by the notation ${ }^{7} F_{J M} X$. It should be emphasized that in the excited states all seven electrons are described by wave functions that differ from their ground-state wave functions.

The position of a $5 d\left(t_{2 g}\right)$ conduction band is determined by the Landé interval rule, and depends only on the quantum number $J$ (ignoring a comparatively small Zeeman energy that arises if a magnetic field is switched on, see also discussion below). Measured from the ground state, it is given by

$$
E_{J}=E_{G}+\frac{1}{2} \lambda_{4 f} J(J+1), \quad J=0, \ldots, 6,
$$

where $E_{G}$ is the band gap and $\lambda_{4 f}$ is the Landé spin-orbit constant for the ${ }^{7} F_{J M}$ state. The total energy of an electronic excitation $F_{J M} X$, in which the electron in state $X$ is described by a Bloch wave vector $\boldsymbol{k}$, will be given by

$$
E_{J X}=E_{J}+\epsilon_{X}(\boldsymbol{k}),
$$

where $\epsilon_{X}(\boldsymbol{k})$ is the dispersion of the $5 d\left(t_{2 g}\right)$ conduction band to which the $X$ state belongs.

The energy-level scheme, displaying the ground state and lowest-energy excited states of the system is shown in Fig. 1. The energy scale applies to EuTe.

Of course, in the real $\mathrm{Eu} X$ system, a continuous energy band is present in the background of the ${ }^{7} F_{J M} X$ states. In the calculations described in this work, no account was taken of the broad $6 s$ and $6 p$ bands that are estimated to overlap at least partially with the ${ }^{7} F_{J M} X$ states, ${ }^{7,8,20-22}$ however, since we are concerned with the band-edge SHG intensity only, it is justifiable to restrict ourselves to the electronic structure shown in Fig. 1, because this level scheme has very successfully described the complex magneto-optical properties observed at the absorption band edge in EuTe and EuSe. ${ }^{9,10}$

\section{SHG IN EUROPIUM CHALCOGENIDES}

If the energy of the incident photon is about half of the energy of the excited states, i.e., $\hbar \omega \sim \frac{1}{2} E_{J X}$, then the theory described in Sec. II applies, and Eq. (5) renders the expected dominant contribution to SHG. Because of inversion symmetry, the ED matrix element [see Eq. (3)] is zero between excited states ${ }^{7} F_{J M}$ and ${ }^{7} F_{J^{\prime} M^{\prime}} X^{\prime}$. These states can be connected by either the QD or MD matrix elements. Both QD and MD elements arise from the same approximation order in the expansion, Eq. (2), i.e., by retaining only up to the linear term in $z$, and therefore are often of the same order of magnitude. However, in the specific scenario studied in this 


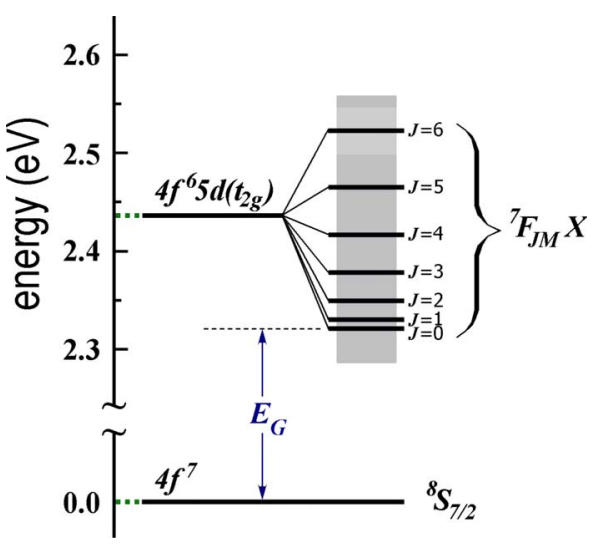

FIG. 1. (Color online) Ground and lowest-energy excited states in $\mathrm{Eu} X$. The distance between the center of the ${ }^{7} F_{J M} X$ for $J=0$ and the ${ }^{8} S_{7 / 2}$ state is taken as the band-gap value $E_{G}$. The splitting between the ${ }^{7} F_{J M}$ manifold is due to the spin-orbit interaction with a spin-orbit constant of $\lambda_{4 f}$. The shaded area indicates the width of the seven $5 d\left(t_{2 g}\right)$ tight-binding overlapping bands. The energy scale applies to antiferromagnetic EuTe at $B=0$, when $E_{G}=E_{G 0}$ $=2.321 \mathrm{eV}$ (Ref. 10) and using $\lambda_{4 f}=9.6 \mathrm{meV}$ (Ref. 10). For $B$ above 7.2 T, the magnetic field imposes ferromagnetic alignment of the spins, and the band gap shrinks to $E_{G}=2.19 \mathrm{eV}$, due to the $d-f$ exchange interaction, and all levels are assumed to redshift rigidly. For intermediate fields, the band gap can be calculated by Eq. (21) (Ref. 19).

work, the QD matrix elements are much smaller than the MD ones. This is because of the factor $E_{a b}$ in the quadrupole matrix element, which is the energy difference between the states involved in the matrix element, and given the small energy spread of the excited states, the electric quadrupole matrix element should be small in comparison to the magnetic dipole matrix element, which does not depend on $E_{a b}$. Indeed, from Eq. (3), the ratio of the amplitude of the QD to the MD second-order polarization can be estimated

$$
\frac{\mathrm{QD}}{\mathrm{MD}}=i \frac{e E_{a b} k_{z} E_{x}}{4 \hbar \omega}\langle a|z x| b\rangle\left[\frac{e B_{y}}{4 m_{0}}\left\langle a\left|L_{y}\right| b\right\rangle\right]^{-1}=i \frac{E_{a b}}{\hbar} m_{0} \frac{\langle a|z x| b\rangle}{\left\langle a\left|L_{y}\right| b\right\rangle} \text {. }
$$

For allowed QD transitions, $\langle a|z x| b\rangle \sim a_{B}^{2}$, where $a_{B}$ is the Bohr radius; for allowed MD transitions, $\left\langle a\left|L_{y}\right| b\right\rangle \sim \hbar$. Substituting these assumptions in Eq. (8) we get

$$
\frac{\mathrm{QD}}{\mathrm{MD}} \sim i \frac{E_{a b}}{\hbar} m_{0} \frac{a_{B}^{2}}{\hbar}=i \frac{E_{a b}}{2 R y},
$$

where $R y=\frac{\hbar^{2}}{2 m_{0} a_{B}^{2}}=13.6 \mathrm{eV}$ is the Rydberg. In our case, $E_{a b}$ represents the energy difference between excited states ${ }^{7} F_{J M} X$, which are spread over an energy band of only a few hundreds of meV (see Fig. 1), thus we finally obtain an estimate for the ratio of the QD matrix element to the MD matrix element

$$
\frac{\mathrm{QD}}{\mathrm{MD}} \sim 0.01
$$

It is thus justifiable to include in the analysis MD matrix elements only. Substituting the excited-states quantum numbers $n$ and $m$ by $J M X$ and $J^{\prime} M^{\prime} X^{\prime}$ in expression (5), the induced second-order polarization component parallel to the polarization vector of the excitation light will be given by

$$
P_{x 0}^{(2)}(2 \omega)=\frac{N e^{3} E_{x} B_{y}}{8 m_{0} \hbar \omega} \sum_{\substack{J M X \\ J^{\prime} M^{\prime} X^{\prime}}} E_{J^{\prime} X^{\prime}} \frac{\left\langle{ }^{8} S_{7 / 2}\left|\sum x_{q}\right|^{7} F_{J M} X\right\rangle\left\langle{ }^{7} F_{J M} X\left|\sum\left(L_{y}\right)_{q}\right|^{7} F_{J^{\prime} M^{\prime}} X^{\prime}\right\rangle\left\langle{ }^{7} F_{J^{\prime} M^{\prime}} X^{\prime}\left|\sum x_{q}\right|^{8} S_{7 / 2}\right\rangle}{\left(E_{J^{\prime} X^{\prime}}-\hbar \omega+i \Gamma\right)\left(E_{J X}-2 \hbar \omega+i \Gamma\right)},
$$

where $N=4 / a^{3}$ is the concentration of $\mathrm{Eu}^{2+}$ in the lattice, $a$ is the parameter of the face-centered cubic lattice of $\mathrm{Eu} X$, and the relaxation between all excited and the ground state is described by a common parameter $\Gamma$. In the above equation, $x_{q}$ and $\left(L_{y}\right)_{q}$ represent the $x$ coordinate and $y$ component of the angular momentum, respectively, of the $q$ th electron, and the sum is taken over all seven electrons involved in the electronic transitions. The SHG mechanism described by Eq. (11) is shown schematically in Fig. 2.

Fundamentally, the spin of the electron excited by light from the ${ }^{8} S_{7 / 2}$ level into the $X$ state is parallel to the spin of the $\mathrm{Eu}^{2+}$ site in which the electron was initially located. This is experimentally demonstrated by the quadratic dependence of the band edge on the intensity of an applied magnetic field. ${ }^{19}$ As demonstrated in Ref. 10, such spin conservation simplifies the problem considerably, because a manyelectron matrix element of an operator acting only on the electronic space coordinates between the ground state ${ }^{8} S_{7 / 2}$ and an excited state ${ }^{7} F_{J M} X$ can be written in terms of the matrix element of a single electron, for instance,

$$
\left\langle{ }^{8} S_{7 / 2}\left|\sum_{q=1}^{7} x_{q}\right|{ }^{7} F_{J M} X\right\rangle=C^{J M}\langle 4 f m|x| X\rangle
$$

where $|4 \mathrm{fm}\rangle$ represents an orbital of an electron in the Coulomb field of the $\mathrm{Eu}^{3+}$ with principle quantum number 4, angular momentum and magnetic quantum numbers $f$ and $m$, respectively; $m=-M+3$, the range of values that need to be taken into account is reduced to $M=0, \ldots, J$, and $C^{J M}$ $=\left(L S M_{L} M_{S} \mid J M\right)$ is a compact representation of ClebschGordan coefficients with $L=S=M_{S}=3, M_{L}=M-3$.

Additionally, 


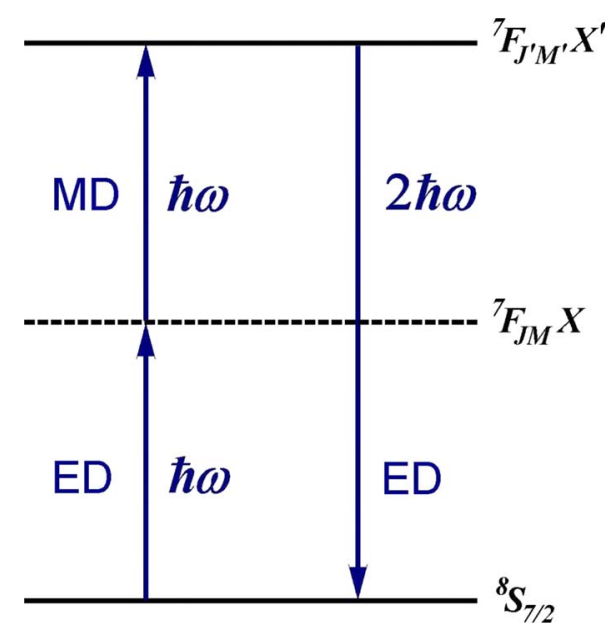

FIG. 2. (Color online) SHG scheme in EuX. The matrix element — electric or magnetic dipole (ED or MD) — involved in the process is indicated.

$$
\begin{aligned}
& \left\langle{ }^{7} F_{J M} X\left|\sum_{q=1}^{7}\left(L_{y}\right)_{q}\right|{ }^{7} F_{J^{\prime} M^{\prime}} X^{\prime}\right\rangle \\
& =\left\langle{ }^{7} F_{J M}\left|\sum_{q=1}^{6}\left(L_{y}\right)_{q}\right|^{7} F_{J^{\prime} M^{\prime}}\right\rangle \delta_{X X^{\prime}}+\left\langle X\left|L_{y}\right| X^{\prime}\right\rangle \delta_{J J^{\prime}} \delta_{M M^{\prime}} .
\end{aligned}
$$

By the same procedure that lead to Eq. (12), i.e., by performing a Clebsch-Gordan expansion, and using spin conservation, the six-electron matrix element $\left\langle{ }^{7} F_{J M}\left|\sum_{q=1}^{6}\left(L_{y}\right)_{q}\right|{ }^{7} F_{J^{\prime} M^{\prime}}\right\rangle$ in Eq. (13) can be substituted by a single-electron leading matrix element

$$
\left\langle{ }^{7} F_{J M}\left|\sum_{q=1}^{6}\left(L_{y}\right)_{q}\right|^{7} F_{J^{\prime} M^{\prime}}\right\rangle=C^{J M} C^{J^{\prime} M^{\prime}}\left\langle 4 f m^{\prime}\left|L_{y}\right| 4 f m\right\rangle,
$$

where $m=-M+3$ and $m^{\prime}=-M^{\prime}+3$.

Substituting Eqs. (12)-(14) in Eq. (11), we obtain the following explicit form for the second-order polarization amplitude, now in terms of one-electron matrix elements

$$
\begin{aligned}
P_{x 0}^{(2)}(2 \omega)= & \frac{N e^{3} E_{x} B_{y}}{8 m_{0} \hbar \omega} \sum_{J M X, J^{\prime} M^{\prime}} E_{J^{\prime} X}\left[C^{J M} C^{J^{\prime} M^{\prime}}\right]^{2} \\
& \times \frac{\left\langle X|x| 4 f m^{\prime}\right\rangle\left\langle 4 f m^{\prime}\left|L_{y}\right| 4 f m\right\rangle\langle 4 f m|x| X\rangle}{\left(E_{J^{\prime} X}-\hbar \omega+i \Gamma\right)\left(E_{J X}-2 \hbar \omega+i \Gamma\right)} \\
& +\frac{N e^{3} E_{x} B_{y}}{8 m_{0} \hbar \omega} \sum_{J M X, X^{\prime}} E_{J X}\left[C^{J M}\right]^{2} \\
& \times \frac{\langle 4 f m|x| X\rangle\left\langle X\left|L_{y}\right| X^{\prime}\right\rangle\left\langle X^{\prime}|x| 4 f m\right\rangle}{\left(E_{J X^{\prime}}-\hbar \omega+i \Gamma\right)\left(E_{J X}-2 \hbar \omega+i \Gamma\right)},
\end{aligned}
$$

where $m=-M+3$ and $m^{\prime}=-M^{\prime}+3$.

In the tight-binding approximation, the $5 d\left(t_{2 g}\right)$ conduction-band Bloch state $X$, of energy $\epsilon_{X}(\boldsymbol{k})$, is given by

$$
X(\boldsymbol{k}, \boldsymbol{r})=\frac{1}{\sqrt{\mathcal{N}}} \sum_{\boldsymbol{R}} e^{i \boldsymbol{R} \cdot \boldsymbol{k}} \phi(\boldsymbol{r}-\boldsymbol{R}),
$$

where $\phi(\boldsymbol{r})$ represents one of the $5 d\left(t_{2 g}\right)$ europium orbitals $\left(d_{x y}, d_{y z}\right.$, or $\left.d_{z x}\right), \boldsymbol{R}$ is the position vector of an Eu atom in the lattice, and $\mathcal{N}$ is the number of lattice sites inside the BornKarmán volume. The energy corresponding to the excitation from the ground state into the $F_{J M} X$ state, $E_{J X}$, is given by Eq. (7). However, it can be anticipated that a rigorous account of the conduction-band energy width will ultimately lead to broadening of the resonances that are characteristic of Eq. (15). In support of this approach is the fact that $\boldsymbol{k}$ is not conserved in the matrix elements, given that the $|4 \mathrm{fm}\rangle$ states are spatially localized at an Eu site, hence matrix elements of $X$ from all over the Brillouin zone will have an equal weight in Eq. (15). We therefore choose a heuristic approach in which in a first step we ignore the conduction-band dispersion. In this first step, the second-order polarization is calculated with a substitution of $X, X^{\prime}, E_{J X}$, and $E_{J^{\prime} X^{\prime}}$ by $\phi, \phi^{\prime}$, $E_{J}$, and $E_{J^{\prime}}$ in Eq. (16), i.e., from

$$
\begin{aligned}
P_{x 0}^{(2)}(2 \omega)= & \frac{N e^{3} E_{x} B_{y}}{8 m_{0} \hbar \omega} \sum_{J M \phi, J^{\prime} M^{\prime}} E_{J^{\prime}}\left[C^{J M} C^{J^{\prime} M^{\prime}}\right]^{2} \\
& \times \frac{\left\langle\phi|x| 4 f m^{\prime}\right\rangle\left\langle 4 f m^{\prime}\left|L_{y}\right| 4 f m\right\rangle\langle 4 f m|x| \phi\rangle}{\left(E_{J^{\prime}}-\hbar \omega+i \Gamma\right)\left(E_{J}-2 \hbar \omega+i \Gamma\right)} \\
& +\frac{N e^{3} E_{x} B_{y}}{8 m_{0} \hbar \omega} \sum_{J M \phi, \phi^{\prime}} E_{J}\left[C^{J M}\right]^{2} \\
& \times \frac{\langle 4 f m|x| \phi\rangle\left\langle\phi\left|L_{y}\right| \phi^{\prime}\right\rangle\left\langle\phi^{\prime}|x| 4 f m\right\rangle}{\left(E_{J}-\hbar \omega+i \Gamma\right)\left(E_{J}-2 \hbar \omega+i \Gamma\right)},
\end{aligned}
$$

where $m=-M+3$ and $m^{\prime}=-M^{\prime}+3$. The conduction-band energy width is taken into account in a second step, by convoluting the second harmonic generated intensity corresponding to Eq. (17) with a density of states characteristic of a $5 d\left(t_{2 g}\right)$ conduction band

$$
I_{\mathrm{SHG}}(2 \hbar \omega) \propto \int d E \mathcal{D}(E-2 \hbar \omega)\left\{\operatorname{Re}\left[P_{x 0}^{(2)}\left(\frac{E}{\hbar}\right)\right]\right\}^{2} .
$$

To calculate matrix elements $\langle 4 f m|x| \phi\rangle,\left\langle 4 f m^{\prime}\left|L_{y}\right| 4 f m\right\rangle$, and $\left\langle\phi\left|L_{y}\right| \phi^{\prime}\right\rangle$, that appear in the expression (17), it is first required that the wave functions for the atomic $|4 \mathrm{fm}\rangle$ state and for the $|\phi\rangle$ state be expressed in the coordinates of the reference frame that is used to describe the propagating light, in which operators $L_{y}$ and $x$ are defined. This can be done using Wigner rotation matrices $D_{m_{1} m_{2}}^{(j)}(\alpha, \beta, \gamma)$ (explicit forms of these matrices are given in Ref. 23). For instance,

$$
\left|4 f m_{1}\right\rangle=\sum_{m_{2}=-3}^{+3} D_{m_{1} m_{2}}^{(3)}\left(\alpha_{S}, \beta_{S}, \gamma_{S}\right)\left|4 f m_{2}\right\rangle,
$$

where $\alpha_{S}, \beta_{S}$, and $\gamma_{S}$ are the Euler angles that transform the reference frame used to describe the $|4 \mathrm{fm}\rangle$ state (in which $z$ direction the is angular momentum quantization axis for the ${ }^{8} S_{7 / 2}$ state) into the reference frame for light propagation (in 
which light travels along z). Similarly, the $\phi$ states must be Euler rotated.

Any orbital $|\phi\rangle$ can be expressed as a linear combination of $\left|5 d m_{1}\right\rangle$ orbitals, i.e., $|\phi\rangle=\Sigma B\left(\phi, m_{1}\right)\left|5 d m_{1}\right\rangle$ [for instance, if $\phi=d_{x y}$, then $B(\phi, 2)=-B(\phi,-2)=\frac{1}{\sqrt{2}}$, all other $B(\phi, m)=0]$. Therefore in the coordinate frame of the propagating light $|\phi\rangle$ is given by

$$
|\phi\rangle=\sum_{m_{1}, m_{2}=-2}^{+2} B\left(\phi, m_{1}\right) D_{m_{1} m_{2}}^{(2)}\left(\alpha_{c}, \beta_{c}, \gamma_{c}\right)\left|5 d m_{2}\right\rangle,
$$

where $\alpha_{c}, \beta_{c}$, and $\gamma_{c}$ are the Euler angles that transform the reference frame of the crystal structure (in which the $z$ direction is parallel to the crystal direction of Miller indices [001]) into the frame for light propagation. (For example, if [110] polarized light travels along [111], and the $\mathrm{Eu}^{2+}$ spins are oriented along $[11 \overline{2}]$, then $\alpha_{c}=\pi / 4, \beta_{c}=\arctan \sqrt{2}, \gamma_{c}$ $=\pi / 2, \alpha_{S}=0, \beta_{S}=\pi / 2$, and $\left.\gamma_{S}=-\pi / 2\right)$.

If we now consider the application of an external constant magnetic field on our EuX system, then the Zeeman interaction will perturb the energy-level scheme shown in Fig. 1. However, we shall disregard any effect the magnetic field may have on the energy levels described in Sec. III, except for a rigid redshift of all excited states in Fig. 1, caused by $B$ dependent the $d$ - $f$ exchange interaction. ${ }^{19}$ The Zeeman energy is orders of magnitude smaller than the energy-level splitting of the ${ }^{7} F_{J} M$ manifold, the characteristic energy width of the $5 d\left(t_{2 g}\right)$ tight-binding band, and the magnetic field dependent $d$ - $f$ exchange interaction energy., 10,18,24 For example, in EuTe the shrinkage of the band gap, due to the $d$ - $f$ exchange interaction energy is $\sim 15 \mathrm{meV} / \mathrm{T},{ }^{19}$ whereas the electronic Zeeman interaction is only $30 \mu \mathrm{eV} / \mathrm{T}$, which we will therefore disregard.

Nonetheless, a magnetic field has a profound effect on the matrix elements that determine the SHG intensities in $\mathrm{EuX}$ because it reorients the spins at the $\mathrm{Eu}^{2+}$ lattice sites. At a given field, at $T=0 \mathrm{~K}$ the orientation of the $\mathrm{Eu}^{2+}$ spins is found by minimizing a molecular-field Hamiltonian that includes the superexchange interaction that favors antiferromagnetic (in EuSe and EuTe) or ferromagnetic (in EuO and EuS) arrangement, the Zeeman interaction which favors alignment of the $\mathrm{Eu}^{2+}$ along the magnetic field direction, and the anisotropy energy which favors the formation of oriented domains at $B=0 .{ }^{24}$ As the $\mathrm{Eu}^{2+}$ spins are reoriented by the magnetic field, this has a direct and dramatic impact on the matrix elements. ${ }^{18}$ As described above, the reference frames in which the $\mathrm{Eu}^{2+}$ and $5 d\left(t_{2 g}\right)$ angular momentum quantization axes are defined, and the quantization frame in which the photon travels, are connected through Euler rotations. The required Euler rotations evidently depend on the spatial orientation of the $\mathrm{Eu}^{2+}$ spins, and this explains why the matrix elements are so sensitive on the direction and intensity of a magnetic field applied externally.

\section{RESULTS AND DISCUSSION}

Using the SHG model described in the previous section, the $\mathrm{SHG}$ at $T=0 \mathrm{~K}$ spectrum was investigated theoretically as a function of an applied magnetic field, $B$, in the Voigt geometry (when $B$ is perpendicular to the exciting light wave vector). Calculations were performed using the well-known energy structure parameters of EuTe, obtained from earlier optical-absorption measurements, ${ }^{10,18,19,24}$ and all parameters were fixed accordingly, as follows. As a function of magnetic field below the saturation value $\left(B \leq B_{\mathrm{SAT}}=7.2 \mathrm{~T}\right)$, the band gap $E_{G}$ was taken to follow a quadratic decrease, and above the saturation it becomes constant ${ }^{19}$

$$
E_{G}=E_{G 0}-J_{d f} S \times\left\{\begin{array}{cl}
\left(\frac{B}{B_{\mathrm{SAT}}}\right)^{2} & \text { if } B<B_{\mathrm{SAT}} \\
1 & \text { if } B \geq B_{\mathrm{SAT}},
\end{array}\right.
$$

where $E_{G 0}=2.321 \mathrm{eV}$ is the zero-field band gap. The $d-f$ exchange constant, $J_{d f} S$, which determines the band-gap dependence on $B,{ }^{19}$ was taken to be $J_{d f} S=130 \mathrm{meV}$; this is the average maximum shift of the absorption lines that compose the dichroic doublet seen in high magnetic fields. ${ }^{9,10}$ The $4 f$ spin-orbit interaction constant was fixed at $\lambda_{4 f}=9.6 \mathrm{meV}{ }^{10}$ The density of states of a given $5 d\left(t_{2 g}\right)$ conduction band was taken to be a Gaussian of full width at half maximum of 100 meV, which approximately corresponds to the energy width of a $5 d\left(t_{2 g}\right)$ conduction band. ${ }^{10,18}$ The only unknown parameter entering the theoretical model is the damping parameter $\Gamma$ [see Eq. (11)], which was taken to be $\Gamma=10 \mathrm{meV}$, which gives an approximate fit to the experimental SHG line shape. ${ }^{13}$

As discussed in the previous section, the orientation of the $\mathrm{Eu}^{2+}$ spins in the crystal play a decisive role on the SHG spectrum, through the matrix elements involved in the process. At $T=0 \mathrm{~K}$ and in the absence of a magnetic field, the

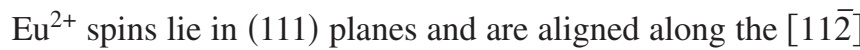
directions. Since there are four equivalent (111) planes, four equivalent so-called $T$ domains may be formed. Within each $T$ domain, there are three equivalent $(11 \overline{2})$ directions, and hence three isoenergetic so-called $S$ domains are formed within each $T$ domain. However, our target was to compare the theory with experiments performed on EuTe thick layer samples (about $1 \mu \mathrm{m}$ thickness) grown by molecular-beam epitaxy (MBE), and therefore only a single $T$ domain parallel to the (111) substrate was included in the calculations, because the other domains of equivalent energy present in isotropic samples are suppressed in MBE samples due to residual strains. ${ }^{25}$ Within the existing $T$ domain, all three $S$ domains are found experimentally to be present in samples grown by $\mathrm{MBE},{ }^{26}$ thus three $S$ domains were included in the calculations.

The orientation of $\mathrm{Eu}^{2+}$ spins in every $S$ domain, for a given magnetic field, was calculated by solving the molecular-field Hamiltonian which included superexchange, Zeeman and anisotropic interactions, as described above and in detail in Refs. 18 and 24. The amplitude of the secondorder-induced polarization generated by every $\mathrm{Eu}^{2+}$ spin orientation was calculated using Eq. (17). The total polarization was obtained by averaging over all three $S$ domains and both spin sublattices. The SHG intensity was calculated using Eq. (18).

Figure 3 shows the calculated SHG spectra as a function of magnetic field, at $0.5 \mathrm{~T}$ intervals, for a magnetic field 


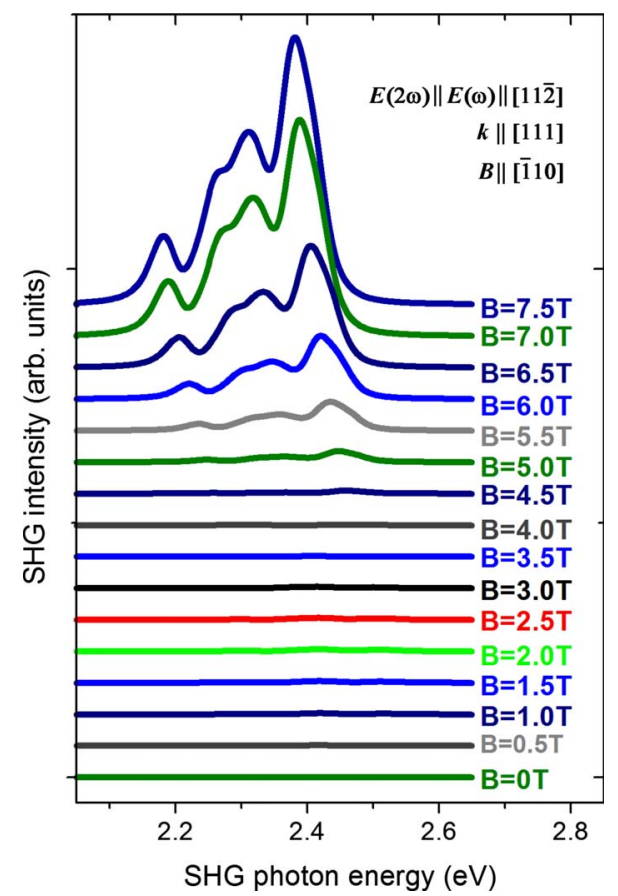

FIG. 3. (Color online) Modeling of magnetic field induced SHG in EuTe at $T=0 \mathrm{~K}$. Calculations were done for $\boldsymbol{E}(2 \omega)\|\boldsymbol{E}(\omega)\|[11 \overline{2}]$, $\boldsymbol{B} \|[\overline{1} 10]$, and $\boldsymbol{k} \|[111]$.

vector $\boldsymbol{B} \|[\overline{1} 10]$, light wave vector $\boldsymbol{k} \|[111]$, excitation light $\boldsymbol{E}(\omega) \|[11 \overline{2}]$, and SHG $\boldsymbol{E}(2 \omega)$ polarization component along the direction of polarization of the excitation light. Theory predicts a drastic increase in the SHG intensity when the applied magnetic field increases, and a saturation of the SHG intensity at 7.2 $\mathrm{T}$ when the magnetic field imposes full ferromagnetic alignment of the lattice spins. Above the saturation field, the SHG intensity does no longer change; the maximum SHG intensity is expected at a photon energy 2.38 $\mathrm{eV}$, which is about $200 \mathrm{meV}$ greater than the EuTe band gap, since $E_{G}(B \geq 7.2 \mathrm{~T})=2.19 \mathrm{eV}$, as given by Eq. (21). The calculated integrated intensity dependence on $B$ is plotted in Fig. 4. In Ref. 13 the SHG intensity, $I_{\mathrm{SHG}}$, dependence on the magnitude of the applied field was examined in the framework of an empirical phenomenological model, in which no specific electronic energy structure is examined, which leads to an SHG intensity proportional to the magnetization squared, $I_{\mathrm{SHG}} \sim M^{2}$. Since below saturation in EuTe $M \propto B,{ }^{27}$ the phenomenological model gives $I_{\mathrm{SHG}} \sim B^{2}$. This contrasts to a more complex theoretical dependence predicted by the microscopical model developed in this work, in which a specific electronic energy structure (Fig. 1) was taken into account. The microscopical model predicts $I_{\mathrm{SHG}}$ remaining nearly zero for up to $B \sim 4 \mathrm{~T}$, and a very steep $I_{\mathrm{SHG}}$ rise above that. Both models fairly describe observations within experimental error, although the phenomenological model is in slightly better quantitative agreement with the data, whereas the microscopic model overestimates the steepness of the $I_{\mathrm{SHG}}$ rise seen in the experimental data.

The estimated position of the SHG maximum above the saturation field, as well as the line shape of the SHG spectrum, can be explained by the electronic structure (Fig. 1)

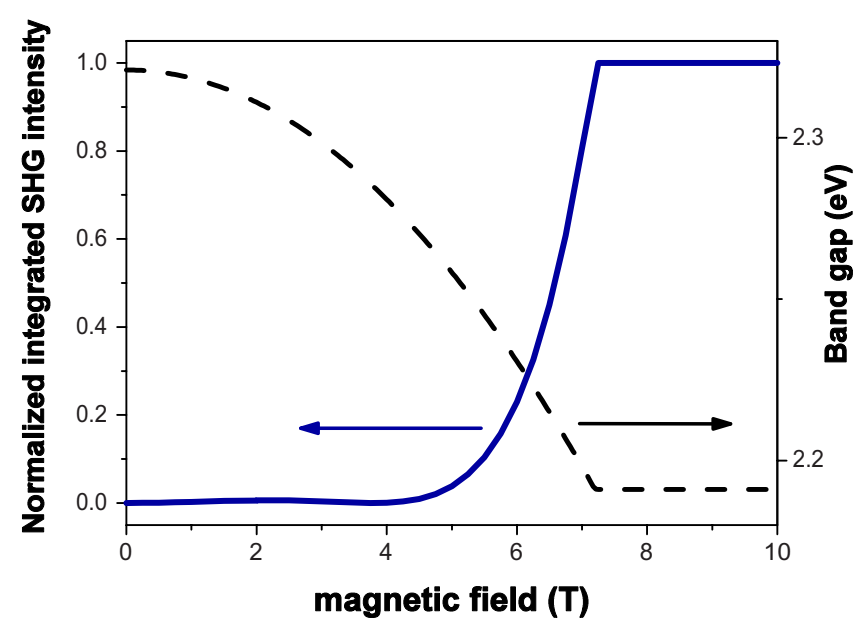

FIG. 4. (Color online) Normalized SHG integrated intensity as a function of the magnetic field (full line). The dashed line shows the band-gap dependence on field in EuTe, as given by Eq. (21).

underlying the theoretical spectra. It so turns out that the SHG signal is most intense when twice the energy of the excitation light is resonant with the states of $J=6$ (see Fig. 1 ), which are the highest energy levels in the model. On the low-energy side, levels with lower $J$ values also contribute to the SHG intensity, although not as strongly as the $J=6 \mathrm{lev}$ els, which explains the line shape obtained.

As a final result, the calculated rotational anisotropy of the SHG intensity for any magnetic field greater than the saturation value of $7.2 \mathrm{~T}$ is shown in Fig. 5. This picture shows that the maximum intensity of the SHG signal is generated at $90^{\circ}$ to the direction of the magnetic field. This result indicates that in the Voigt geometry presently investigated the largest magnetic dipole oscillating vector that exciting light may induce between excited states $F_{J M} X$ and $F_{J^{\prime} M^{\prime}} X^{\prime}$ is oriented along the direction of the external magnetic field.

The theoretical results shown in Figs. 3-5 are in very good agreement with recent experimental results for EuTe. ${ }^{13}$ The calculated position of the SHG maximum signal is at

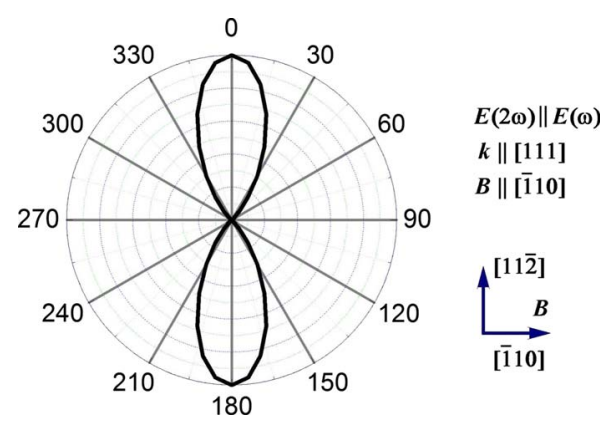

FIG. 5. (Color online) Polar plot of the calculated SHG anisotropic intensities in EuTe. The angle shown is measured between the $[11 \overline{2}]$ crystalline direction and the polarization vector of the incoming light, which traveled along the [111] direction. The magnetic field was directed along [ $\overline{1} 10]$. The calculations shown were done for the SHG intensity polarized parallel to the incident light. The distance from the origin represents the intensity of the signal for a given angle. 
$2.38 \mathrm{eV}$, whereas experimentally it is seen at $2.40 \mathrm{eV} .^{13}$ The width at half maximum of the theoretical line is $\sim 160 \mathrm{meV}$, which compares to the experimental result of $\sim 100 \mathrm{meV} .{ }^{13}$ The theoretical line shape, described by a slow onset of SHG at the low-energy side, and a well-defined cutoff at the highenergy side of the maximum SHG, is also in good agreement with the experimental one. ${ }^{13}$ Finally the directional dependence of the SHG found by the model is in almost perfect agreement with measurements. ${ }^{13}$

It should be noticed, however, that although the coincidence between SHG theory and experiment in EuTe is striking, the agreement is not complete. The theoretical spectrum shows a number of sharp lines at the low-energy side of the SHG maximum that are not observed experimentally. This discrepancy can be attributed to the simplicity of the theory used here. For example, we have neglected the spin-orbit interaction within the $5 d\left(t_{2 g}\right)$ levels, ${ }^{21}$ which should contribute to washing out the finest theoretical features seen in Fig. 3(a). We have also disregarded a background density of states, which is observed, for instance, in absorption measurements, which display an increasing optical density toward increasing energies. ${ }^{9}$ Nevertheless, because of the good agreement between the model and the experiment, which shows no SHG at the high-energy side of the maximum, it follows that the background density of states effectively irrelevant in the SHG process in EuTe, at least in the photon energy range investigated in this work.

Another aspect that deserves to be commented upon is the evident correlation between the SHG intensity and the magnetization in EuTe. The magnetization of EuTe increases linearly with applied magnetic field, ${ }^{27}$ whereas the SHG intensity increase is superlinear, ${ }^{13}$ which is well reproduced by the model presented in this work (see Fig. 4). The correlation between magnetization and SHG intensity is explained in the framework of the present model as follows. As discussed above, as far as SHG is concerned, it is sufficient to assume that the sole effect of an external magnetic field on the sample is to reorient the $\mathrm{Eu}^{2+}$ spins, and ultimately align them with the external magnetic field, which is when both SHG and magnetization are largest. The model predicts a SHG intensity that increases superlinearly with $B$, which agrees with experiment ${ }^{13}$ and which contrasts to the linear increase in the magnetization.

\section{CONCLUSION}

In conclusion, we have developed a theoretical model to describe the magnetic field induced SHG process in $\mathrm{Eu} X$, which is a centrosymmetric system that under usual circumstances does not generate second harmonic light. Calculations were presented for EuTe. In the Voigt geometry, theory predicts a steep superlinear dependence of SHG intensity on magnetic field. The SHG process is explained by a magnetic dipole coupling of the excited states. For antiferromagnetic alignment, when the two sublattice spins point in opposite directions, the two sublattices contribute with roughly opposite phases to the second-order polarization, and SHG is very weak. However, if the lattice spins can be made to become aligned with each other, and this can be achieved by applying an external magnetic field, then the magnetic dipole coupling contributions from the two sublattices become in phase with one another, which causes a drastic increase in the SHG intensity. From the symmetry point of view, it is reasoned that when an external magnetic field causes full alignment of the $\mathrm{Eu}^{2+}$ spins with itself, although the crystallographic point group of the structure remains $m 3 m,{ }^{14}$ the symmetry of the electronic charge distribution that gives rise to the SHG process is lowered, and magnetic dipole induced SHG becomes allowed.

These results are in agreement with SHG experiments in EuTe. ${ }^{13}$ Theoretical calculations describe faithfully the experimental data in respect to the position of the SHG maximum, the SHG spectrum line shape, the intensity dependence on external magnetic field, and in respect to the rotational anisotropy of the SHG signal.

The model was based on a simple energy-level scheme that is common to all europium chalcogenides. The same electronic structure has very successfully described such a fundamental property as the absorption band edge in EuTe and EuSe, including a huge magnetic circular dichroism in an energy interval of about $400 \mathrm{meV}$ near the band gap. ${ }^{9,10,24}$ Obviously, due to the similarity of their band-edge optical-absorption ${ }^{9}$ and SHG spectra, ${ }^{13}$ the same theoretical modeling used here for SHG in EuTe is applicable also to EuSe, and could be extended to the complete family of europium chalcogenides, given the similarity in their bandedge optical properties, ${ }^{8}$ which are associated to the same near-gap electronic structure as the one used in this work, despite the fact each member of the $\mathrm{EuX}$ family have quite different magnetic properties.

\section{ACKNOWLEDGMENTS}

Illuminating discussions with R. V. Pisarev, V. V. Pavlov, D. R. Yakovlev, B. Kaminski, and M. Lafrentz are gratefully acknowledged. This work was supported by funding agencies FAPESP, CNPq, and DAAD.
${ }^{1}$ J. O. Dimmock, IBM J. Res. Dev. 14, 301 (1970).

${ }^{2}$ T. S. Santos, J. S. Moodera, K. V. Raman, E. Negusse, J. Holroyd, J. Dvorak, M. Liberati, Y. U. Idzerda, and E. Arenholz, Phys. Rev. Lett. 101, 147201 (2008).

${ }^{3}$ A. Schmehl, V. Vaithyanathan, A. Herrnberger, S. Thiel, C. Richter, M. Liberati, T. Heeg, M. Röckerath, L. F. Kourkoutis, S.
Mühlbauer, P. Böni, D. A. Muller, Y. Barash, J. Schubert, Y. Idzerda, J. Mannhart, and D. G. Schlom, Nature Mater. 6, 882 (2007).

${ }^{4}$ S. Methfessel, IEEE Trans. Magn. 1, 144 (1965).

${ }^{5}$ T. Kasuya and A. Yanase, Rev. Mod. Phys. 40, 684 (1968).

${ }^{6}$ W. Reim and J. Schoenes, Ferromagnetic Materials (North- 
Holland, Amsterdam, 1990), Vol. 5, p. 133.

${ }^{7} \mathrm{P}$. Wachter, Handbook on the Physics and Chemistry of Rare Earth (North-Holland, Amsterdam, 1979), Vol. 1, p. 507.

${ }^{8}$ A. Mauger and C. Godart, Phys. Rep. 141, 51 (1986).

${ }^{9}$ A. B. Henriques, A. Wierts, M. A. Manfrini, G. Springholz, P. H. O. Rappl, E. Abramof, and A. Y. Ueta, Phys. Rev. B 72, 155337 (2005).

${ }^{10}$ A. B. Henriques, M. A. Manfrini, P. H. O. Rappl, and E. Abramof, Phys. Rev. B 77, 035204 (2008).

${ }^{11}$ M. Fiebig, V. V. Pavlov, and R. V. Pisarev, J. Opt. Soc. Am. B 22, 96 (2005).

${ }^{12}$ M. Fiebig, T. Lottermoser, D. Frölich, A. V. Goltsev, and R. V. Pisarev, Nature (London) 419, 818 (2002).

${ }^{13}$ B. Kaminski, M. Lafrentz, R. V. Pisarev, D. R. Yakovlev, V. V. Pavlov, V. A. L. A. B. Henriques, G. Springholz, G. Bauer, E. Abramof, P. H. O. Rappl, and M. Bayer, Phys. Rev. Lett. 103, 057203 (2009).

${ }^{14}$ R. R. Birss, Symmetry and Magnetism (North-Holland, Amsterdam, 1967).

${ }^{15}$ A. S. Davydov, Quantum Mechanics (Pergamon, New York, 1976).

${ }^{16}$ R. W. Boyd, Nonlinear Optics (Academic, New York, 2003).

${ }^{17}$ Y. R. Shen, The Principle of Nonlinear Optics (Wiley, New
York, 1984).

${ }^{18}$ A. B. Henriques, G. D. Galgano, and E. Abramof, J. Phys.: Condens. Matter 20, 255209 (2008).

${ }^{19}$ L. K. Hanamoto, A. B. Henriques, N. F. Oliveira, P. H. O. Rappl, E. Abramof, and A. Y. Ueta, J. Phys.: Condens. Matter 16, 5597 (2004).

${ }^{20}$ T. Kasuya, J. Magn. Magn. Mater. 195, 141 (1999).

${ }^{21}$ G. Guntherodt, P. Wachter, and D. M. Imboden, Phys. Kondens. Mater. 12, 292 (1971).

${ }^{22}$ G. Guntherodt, Phys. Condens. Matter 18, 37 (1974).

${ }^{23}$ D. A. Varshalovich, A. N. Moskalev, and V. K. Khersonskii, Quantum Theory of Angular Momentum (World Scientific, Singapore, 1988).

${ }^{24}$ A. B. Henriques, G. D. Galgano, B. Díaz, P. H. O. Rappl, and E. Abramof, J. Phys.: Condens. Matter 19, 406234 (2007).

${ }^{25}$ H. Kepa, G. Springholz, T. M. Giebultowicz, K. I. Goldman, C. F. Majkrzak, P. Kacman, J. Blinowski, S. Holl, H. Krenn, and G. Bauer, Phys. Rev. B 68, 024419 (2003).

${ }^{26}$ B. Díaz, E. Granado, E. Abramof, P. H. O. Rappl, V. A. Chitta, and A. B. Henriques, Phys. Rev. B 78, 134423 (2008).

${ }^{27}$ A. B. Henriques, L. K. Hanamoto, E. T. Haar, E. Abramof, A. Y. Ueta, and P. H. O. Rappl, Int. J. Mod. Phys. B 18, 3813 (2004). 